\title{
New algebraic and geometric constructs arising from Fibonacci numbers
}

\section{In honor of Masami Ito}

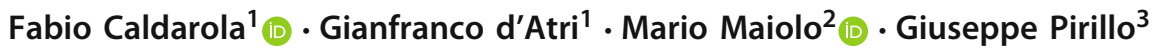

Published online: 29 August 2020

(c) The Author(s) 2020

\begin{abstract}
Fibonacci numbers are the basis of a new geometric construction that leads to the definition of a family $\left\{C_{n}: n \in \mathbb{N}\right\}$ of octagons that come very close to the regular octagon. Such octagons, in some previous articles, have been given the name of Carboncettus octagons for historical reasons. Going further, in this paper we want to introduce and investigate some algebraic constructs that arise from the family $\left\{C_{n}: n \in \mathbb{N}\right\}$ and therefore from Fibonacci numbers: From each Carboncettus octagon $C_{n}$, it is possible to obtain an infinite (right) word $W_{n}$ on the binary alphabet $\{0,1\}$, which we will call the $n$th Carboncettus word. The main theorem shows that all the Carboncettus words thus defined are Sturmian words except in the case $n=5$. The fifth Carboncettus word $W_{5}$ is in fact the only word of the family to be purely periodic: It has period 17 and periodic factor 00010010001001001 . Finally, we also define a further word $W_{\infty}$ named the Carboncettus limit word and, as second main result, we prove that the limit of the sequence of Carboncettus words is $W_{\infty}$ itself.
\end{abstract}

Keywords Approximate constructions · Computing on words · Fibonacci numbers · Sturmian words · Mechanical sequences · Limit of words · Isogonal polygons

Communicated by Yaroslav D. Sergeyev.

M. Ito is one of the world's leading experts on combinatorial and algebraic properties of words and languages.

Fabio Caldarola

caldarola@mat.unical.it

Gianfranco d'Atri

datri@mat.unical.it

Mario Maiolo

mario.maiolo@unical.it

Giuseppe Pirillo

pirillo@math.unifi.it

1 Department of Mathematics and Computer Science, Università della Calabria, Cubo 31/A, Arcavacata di Rende, CS 87036, Italy

2 Department of Environmental Engineering, Università della Calabria, Cubo 42/B, Arcavacata di Rende, CS 87036, Italy

3 Department of Mathematics and Computer Science 'U. Dini', Università di Firenze, viale Morgagni 67/A, Firenze 50134, Italy

\section{Introduction}

In this paper, we associate with each Fibonacci number $F_{n}, n \geq 1$, a geometric construct $C_{n}$ and then an algebraic object $W_{n}$, obtaining simultaneously three sequences $\left\{F_{n}\right\}_{n},\left\{C_{n}\right\}_{n}$ and $\left\{W_{n}\right\}_{n}$, where the first one is the well-known sequence of integers, but the last two are new sequences not of numbers but of geometrical and algebraic objects, respectively. In particular, $\left\{C_{n}\right\}_{n}$ is a sequence of octagons very close to a regular one and $\left\{W_{n}\right\}_{n}$ is a sequence of infinite right words on the binary alphabet $\{0,1\}$. The reasons that led us to give them the name of Carboncettus octagons and words come, as we will see, from far away.

Prato is a Tuscan city located $17 \mathrm{~km}$ northwest of Florence. With its 200,000 inhabitants, Prato is the third largest city in central Italy, after Rome and Florence. The Cathedral of Prato, dedicated to the first Christian martyr Saint Stephen, is a jewel of Romanesque architecture of international appeal: It has ancient roots dating back to at least the sixth century $\mathrm{AD}$ and has undergone numerous renovations and modifications over the years, including, very important, 
those around the eleventh century. The current lateral portal of the Cathedral (Fig. 1) seems to have been the main portal at that time, and, in recent years, it has attracted the attention of various scholars, including several mathematicians, in an attempt to explain some figures inlaid in marble on the sides of the portal. It seems that on the right jamb of the portal, the regularity, symmetry and the subject of the figures recall the divine perfection and completeness, while on the left jamb, some almost regular figures seem to symbolize the limits of human nature, which is made, yes, in the likeness of God, but which is not divine, nor perfect as the divine one (for a deeper study into the rich symbolisms behind the representations on the sides of the portal, the reader can see Pirillo (2017a) and the references therein).

G. Pirillo was fascinated by this portal and its typically medieval symbolism from the first time he saw it in the 70s. Above all, the two inlays with octagonal (or apparently octagonal) symmetry placed at the top of each jamb attracted him very much. The one on the right seems to be based on a perfect regular octagon, while the one on the left seems to allude to a different geometric construction of an octagon that uses two concentric circumferences. Pirillo recently noted that if the radii of the circumferences are equal to two Fibonacci numbers with indices of the same parity and consecutive, then, by means of a simple construction that uses two pairs of parallel tangents to the internal circumference, and perpendicular to each other, one obtains an octagon that is indistinguishable from a regular one, but which is not itself perfectly regular. ${ }^{1}$ In few words, this can be viewed as the discovery of a beautiful approximate method to construct a (not perfectly precise) regular octagon.

It is also very important to note the following fact: The Liber Abaci by Leonardo Pisano called Fibonacci, where the $\left\{F_{n}\right\}_{n}$ series bearing his name appears for the first time, dates back to 1202 AD, while the portal of the Cathedral of Prato, for as seen today, dates back to the previous century and seems to be the work of Carboncettus Marmoriarius, very active in the twelfth century in those places. If the use of Fibonacci numbers in the architecture of the portal in Prato were to be confirmed by other studies, this would be a fact of enormous importance for two main reasons:

\footnotetext{
1 That is, a couple of Fibonacci numbers of the kind $\left(F_{n}, F_{n+2}\right)$ gives rise to the Carboncettus octagon $C_{n}$, as better explained in Sect. 2 with more geometric details. All the Carboncettus octagons are very close to a regular one, with the exception of the first three or at most four elements of the family $\left\{C_{n}: n \in \mathbb{N}\right\}$ (see, for instance, Fig. 4 which displays the already not great difference between a regular octagon and $C_{2}$; see, moreover, Eqs. (2), (3), (4) and Table 1 which collect some metric data of these initial, "special" elements, and see Pirillo's papers (Pirillo 2017a, 2018) for the first observations of such a (suspected) construction by Carboncettus himself).
}

- This would mean that the Fibonacci numbers were known at least a few decades before $1202{ }^{2}$

- The use of mathematical languages or tools in architecture to express concepts and ideas related to medieval (not only religious) symbolism seems not to have precedents before the twelfth century.

In the last two years, Pirillo has discussed many times the content of Pirillo (2017c) with the other authors of this work, and this has led to the article Caldarola et al. (2020c) on "The sequence of Carboncettus octagons." In this paper instead, the main novelty is represented by the definition of an algebraic object for each octagon $C_{n}$ and, hence, for each Fibonacci number $F_{n}$, it is the right infinite word $W_{n}$ on the binary alphabet $\{0,1\}$ that will be precisely defined in Sect. 3 as a lower cutting sequence related to the extension of the height of a triangle which constitutes the octagon $C_{n}$ and will be named the nth Carboncettus word. Such an idea to introduce these algebraic objects follows an intuition of the first author about a peculiarity of the word $W_{5}$ as expressed in Theorem 1: All the words $W_{n}$ result to be Sturmian words except just in the single case $W_{5}$ where we obtain a periodic word with period 17 and periodic factor 00010010001001001 .

After the present introduction, this paper is organized into three central sections: the first two of which take up their titles from and deal with the mentioned geometric and algebraic aspects, respectively. In Sect. 2, we will introduce the geometric constructions, arising from Fibonacci numbers, that lead us to the family of Carboncettus octagons. In Sect. 3 instead, we will define, give some examples of and investigate the algebraic constructs obtained through the $\left\{C_{n}\right\}_{n}$ family from the Fibonacci series as well: They are the Carboncettus words $W_{n}$, and Theorem 1, mentioned above, states our first main result on them. Finally, in Sect. 4 we will introduce the Carboncettus limit word $W_{\infty}$ and, as second main result of the paper, we will prove in Theorem 2 that the limit of the sequence of Carboncettus words is the Carboncettus limit word.

We inform the reader that we have tried to make this text self-contained and therefore suitable for reading even by

\footnotetext{
2 We notice that P. Armienti and A. Albano, in Armienti (2016) and Albano (2015), respectively, investigated a lunette decoration of the church of San Nicola in Pisa (Tuscany, IT), and they found the use of the first Fibonacci numbers until to $F_{10}=55$ and of the golden section. The dating of the decoration and the building is controversial: According to some sources, it dates back to $1281 \mathrm{AD}$, according to other opinions, to the first decades of the thirteenth century. For completeness of information, we point out that G. Pirillo has recently proposed an audacious thesis about the real discovery of Fibonacci numbers: He argues that some members of the Pythagorean School, very active in Kroton (today's city of Crotone, in southern Italy) and in Magna Graecia in the sixth, fifth and fourth centuries BC, must have necessarily met and considered the sequence $\left\{F_{n}\right\}_{n}$ during their mathematical speculations (see, for example, Pirillo 2017b, c, 2018).
} 
Fig. 1 The side portal of the Cathedral of Prato, dating back to the twelfth century AD. The comparison between the two tarsias located at the top of the jambs inspired the (hypothesis on the) construction of the Carboncettus octagon

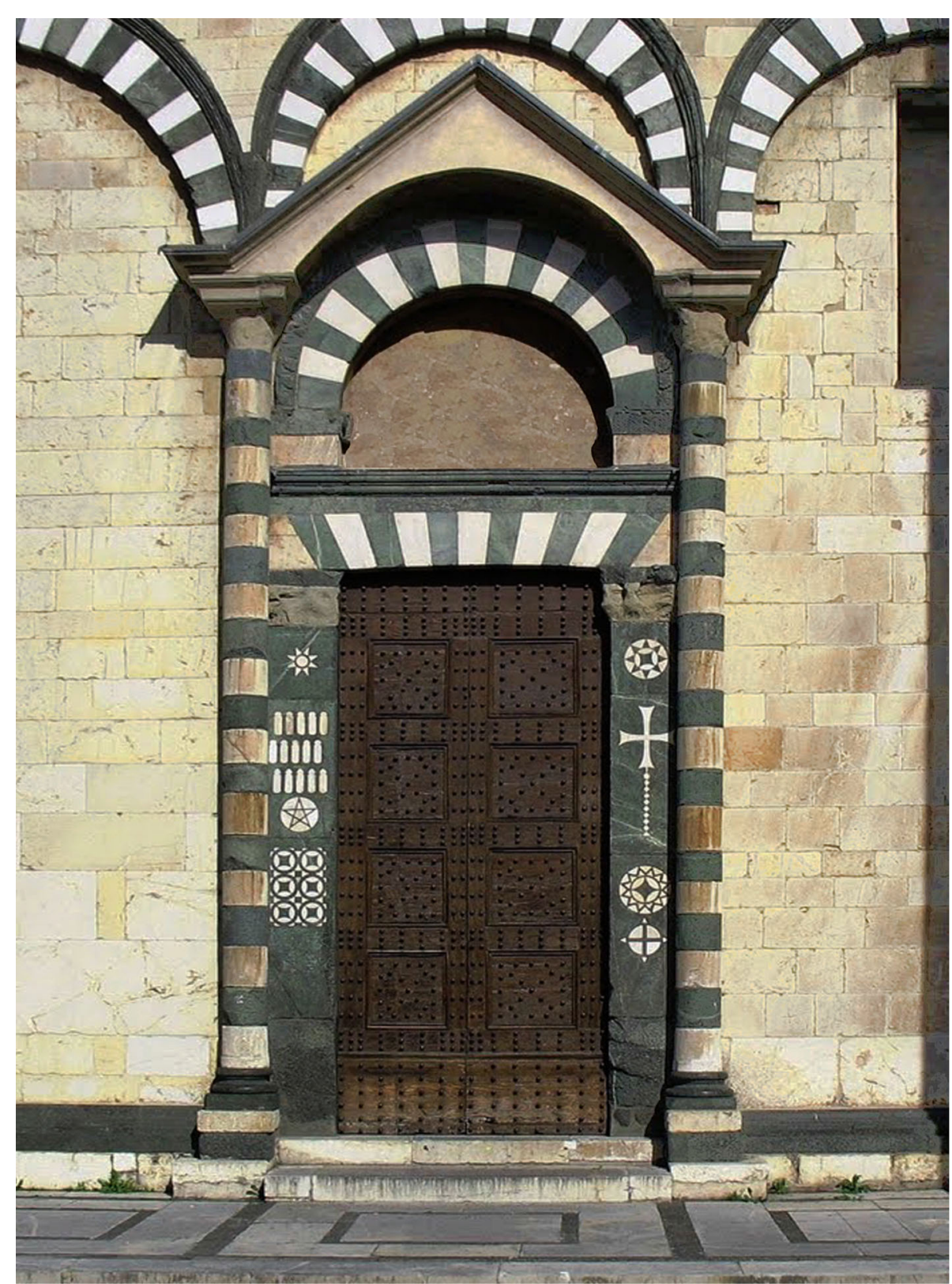

those who are not specialists in the field. Only in the proof of Theorem 1, in fact, we do use three results that are not proven here.

As for notations, we use $\mathbb{N}$ for the set of positive integers and $\mathbb{N}_{0}$ for $\mathbb{N} \cup\{0\}$. A sequence is denoted by $\left\{a_{n}\right\}_{n \in \mathbb{N}}$, $\left\{a_{n}\right\}_{n}$, or sometimes simply $\left\{a_{n}\right\}$. If $A, B, C$ are three points on the plane, we let $A B$ denote the line segment with endpoints $A, B$, by $|A B|$ its length and by $\measuredangle A B C$ the measure (in radians or degrees) of the angle with vertex in $B .^{3}$

3 We follow the usual convention of plane geometry that $0 \leq \measuredangle A B C=$ $\measuredangle C B A \leq \pi$ is always meant.

\section{New geometric constructions from Fibonacci numbers: the Carboncettus family of octagons}

We recall that Fibonacci numbers are defined by $F_{0}:=$ $0, F_{1}:=1$, and

$F_{n}:=F_{n-2}+F_{n-1}$

for all $n \in \mathbb{N}, n \geq 2$; in this way, we obtain the well-known sequence

$0,1,1,2,3,5,8,13,21,34,55,89$,

144, 233, 377, 610, 987, 1597, 2584, etc. 
Fig. 2 The construction of the first Carboncettus octagon $C_{1}$

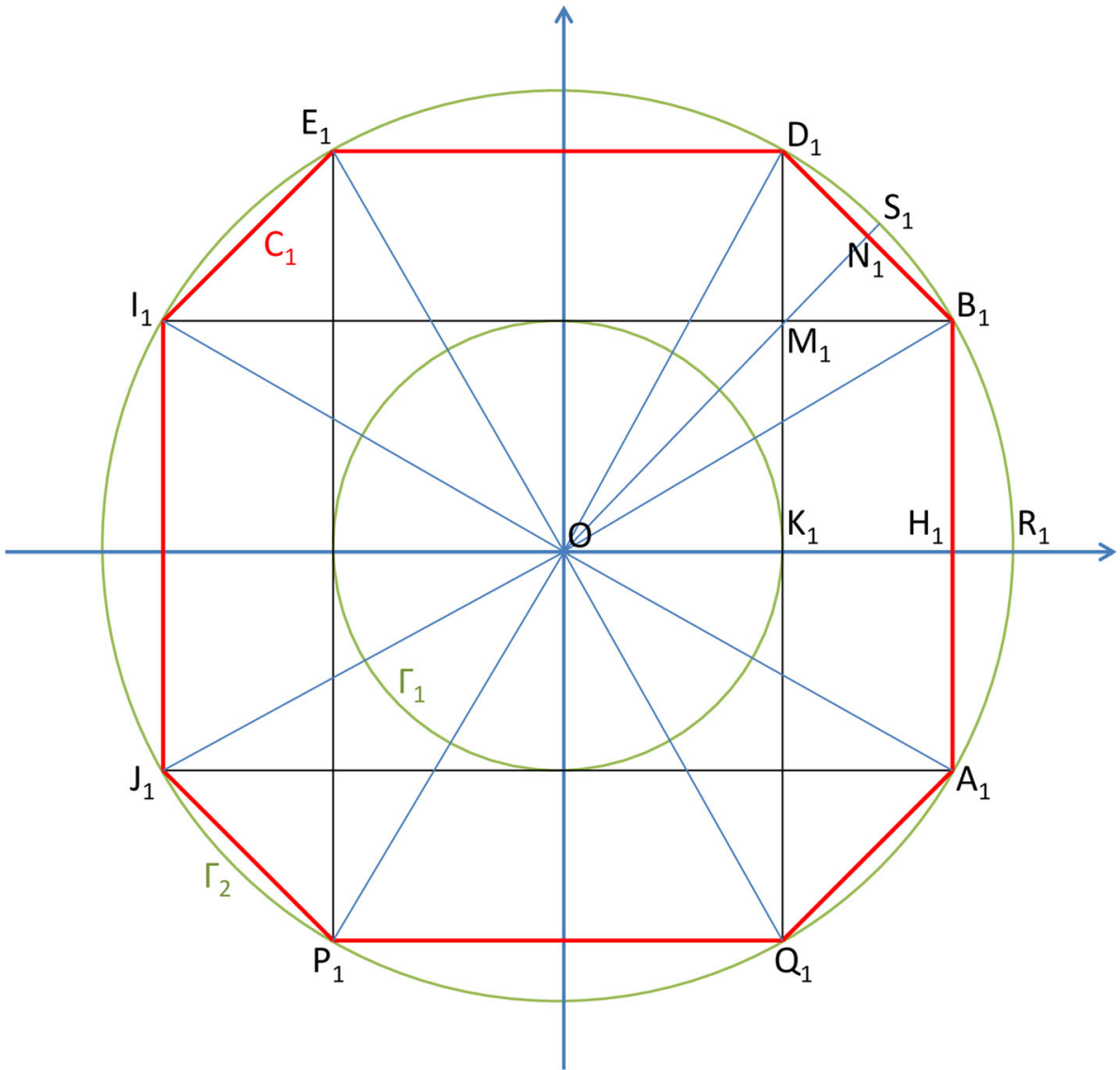

For every real number $r>0$, we let $\Gamma_{r}$ denote the circumference in the plane $\mathbb{R}^{2}$, centered at the origin and with radius $r$. For every $n \in \mathbb{N}$, we consider the couple of Fibonacci numbers $\left(F_{n}, F_{n+2}\right)$ and the circumferences $\Gamma_{F_{n}}, \Gamma_{F_{n+2}}$, with radii $F_{n}$ and $F_{n+2}$, respectively. In the following, we will often refer to $\Gamma_{F_{n}}$ as the internal circumference and to $\Gamma_{F_{n+2}}$ as the external one. When $n=1, \Gamma_{F_{1}}=\Gamma_{1}$ and $\Gamma_{F_{3}}=\Gamma_{2}$ appear in green in Fig. 2, instead Fig. 3 depicts the case for general $n \geq 1$. We then draw a pair of horizontal tangents to the internal circumference $\Gamma_{F_{n}}$ through the two intersection points with the $y$-axis and a pair of vertical tangents through the two intersection points with the $x$-axis. These four straight lines, pairwise parallel or orthogonal, intersect the external circumference $\Gamma_{F_{n+2}}$ at eight points that we denote, starting from the first quadrant and proceeding counterclockwise, by $B_{n}, D_{n}, E_{n}, I_{n}, J_{n}, P_{n}, Q_{n}, A_{n}$ (Fig. 3). The $n$th Carboncettus octagon, denoted by $C_{n}(n \geq 1)$, is the one obtained by drawing the polygonal through the points

$A_{n}, B_{n}, D_{n}, E_{n}, I_{n}, J_{n}, P_{n}, Q_{n}, A_{n}$,

and it is represented in red in Fig. 3.

Remark 1 Every Carboncettus octagon $C_{n}$ is obviously a cyclic polygon, that is, a polygon whose vertices all lie on the same circumference. But not only, $C_{n}$ is, more precisely, an isogonal octagon for all $n \geq 1$. An isogonal or vertextransitive polygon is a polygon whose symmetry group acts transitively on the set of all its vertices. Therefore, an isogonal polygon is equiangular, cyclic and has at most two different alternating side lengths.

A very interesting property of the Carboncettus sequence $\left\{C_{n}\right\}_{n \in \mathbb{N}}$ is that all its elements, with the exception of the first three or at most the first four, are completely indistinguishable from a regular octagon. See, for instance, Fig. 4 which compares the octagon $C_{2}$ with a regular one inscribed in the same circumference $\Gamma_{F_{4}}=\Gamma_{3}$ : They are yet not too far one from the other.

Example 1 (The octagon $C_{1}$ ) The first Carboncettus octagon is obtained starting from the circumferences $\Gamma_{F_{1}}=\Gamma_{1}$ and $\Gamma_{F_{3}}=\Gamma_{2}$ (Fig. 2), and the resulting isogonal octagon $C_{1}$ has very particular characteristics. For instance, all the angles appearing in Fig. 2 are commensurable, because their measures are integer multiples of

$\measuredangle B_{1} O N_{1}=\frac{\pi}{12}=15^{\circ}$.

Moreover, $C_{1}$ results composed of four equilateral triangles congruent to $A_{1} O B_{1}$ and four isosceles triangles congruent 
Fig. 3 The construction of the $n$th Carboncettus octagon $C_{n}$ for a general $n \geq 1$. In the picture, to avoid small and hard to read nested subscripts, we write $\Gamma^{\prime}$ and $\Gamma^{\prime \prime}$ instead of $\Gamma_{F_{n}}$ and $\Gamma_{F_{n+2}}$, respectively

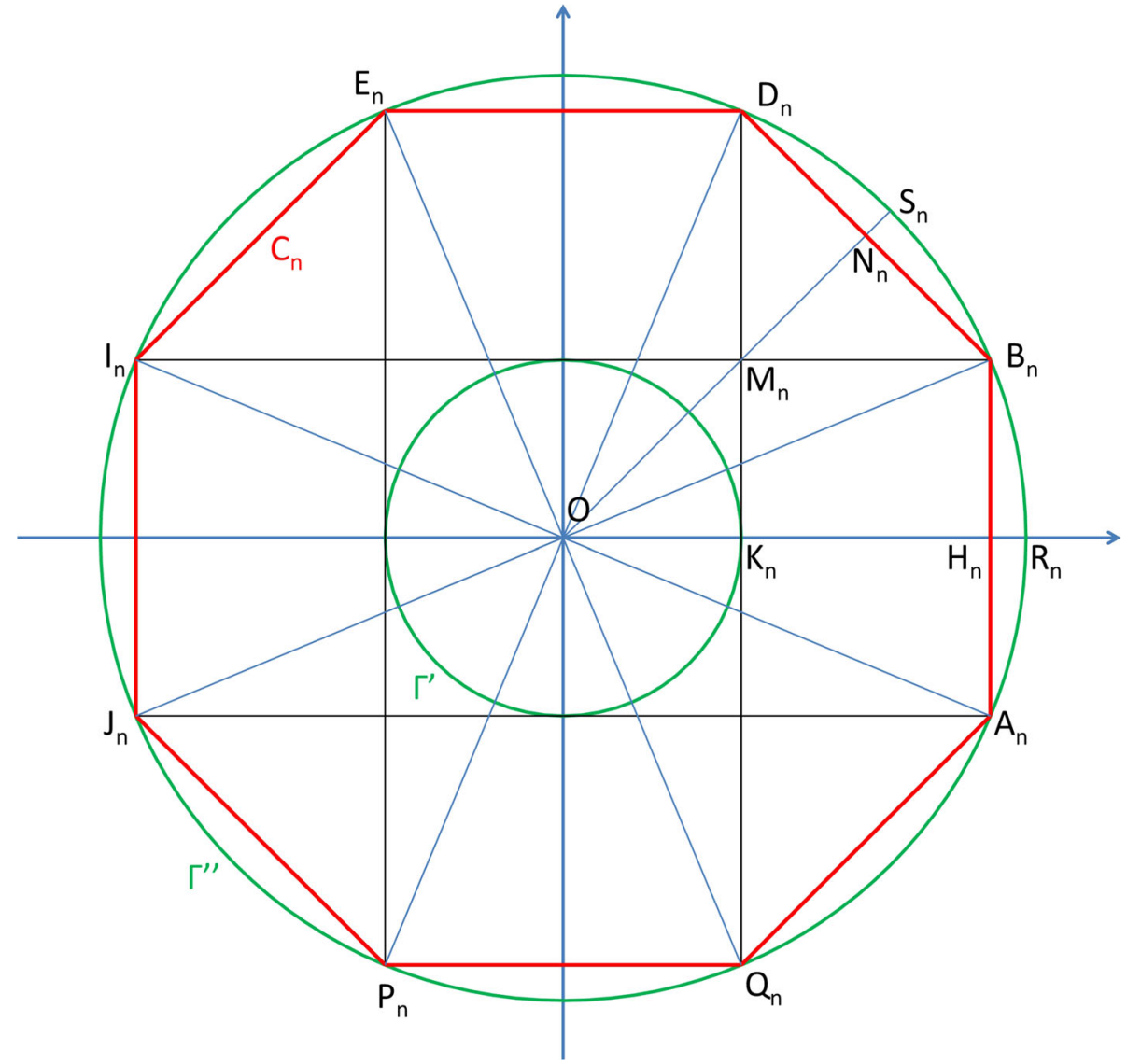

to $B_{1} O D_{1}$. The lengths of their sides and heights are

$$
\begin{aligned}
& \left|A_{1} B_{1}\right|=\left|O A_{1}\right|=2,\left|O H_{1}\right|=\left|K_{1} D_{1}\right|=\sqrt{3} \\
& \left|B_{1} D_{1}\right|=\sqrt{6}-\sqrt{2}, \quad\left|O N_{1}\right|=\frac{\sqrt{6}+\sqrt{2}}{2}
\end{aligned}
$$

and the widths of the involved angles trivially are

$$
\begin{aligned}
& \measuredangle A_{1} O B_{1}=\measuredangle O B_{1} A_{1}=\frac{\pi}{3}=60^{\circ}, \\
& \measuredangle B_{1} O D_{1}=\measuredangle H_{1} O B_{1}=\frac{\pi}{6}=30^{\circ}, \\
& \measuredangle D_{1} B_{1} O=5 \frac{\pi}{12}=75^{\circ} .
\end{aligned}
$$

Note, for example, that, unlike the widths of the angles, all the lengths expressed in (2) are incommensurable in pairs. Lastly, perimeter and area of $C_{1}$ are as follows:

$\operatorname{Perim}\left(C_{1}\right)=8+4 \sqrt{6}-4 \sqrt{2}$,

$\operatorname{Area}\left(C_{1}\right)=4+4 \sqrt{3}$.

Example 2 (The octagons $C_{2}, C_{3}$ and $C_{4}$ ) The construction of the second Carboncettus octagon is based on the circumferences $\Gamma_{1}$ and $\Gamma_{3}$, whose radii are $F_{2}=1$ and $F_{4}=3$, respectively. The obtained octagon $C_{2}$ is drawn in black in Fig. 4, where it is compared with a red regular octagon inscribed in the same circumference $\Gamma_{3}$. Some metric data concerning the octagon $C_{2}$ are listed in the second column of Table 1. Then, the third and the fourth column of the table do the same for the Carboncettus octagons $C_{3}$ and $C_{4}$, respectively.

Much can be said, and there is much to study and investigate on the Carboncettus family of octagons $\left\{C_{n}: n \in \mathbb{N}\right\}$, but we will do it elsewhere because, as anticipated in the Introduction, we must now move on to the next section to introduce new algebraic constructs.

\section{New algebraic constructs from Fibonacci numbers: the Carboncettus words $W_{n}$}

The main reference for the general setting of this section is Lothaire (2002), but the reader can also see Lothaire (1983) and Berstel and Perrin (2007) for an essay on the recent origin of the field called combinatorics on words.

First of all, we need to recall a generalization of (1): A sequence $\left\{G_{n}\right\}_{n \in \mathbb{N}_{0}}$ of integers (but also real or complex num- 


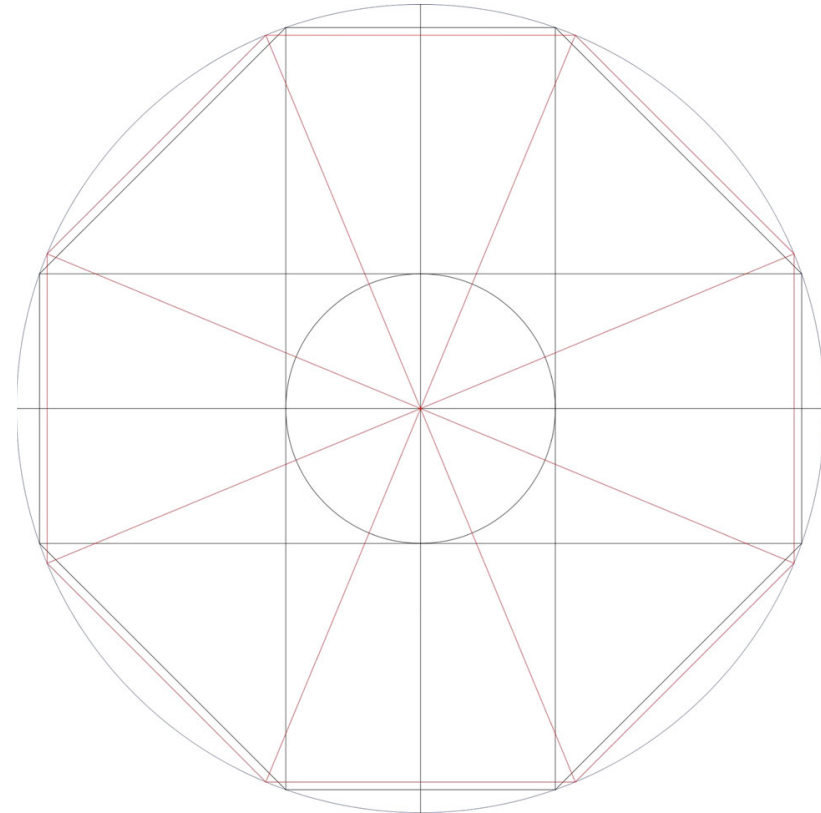

Fig. 4 The octagon $C_{2}$, the second element of the Carboncettus sequence, is drawn in black starting from the circumferences $\Gamma_{F_{2}}=\Gamma_{1}$ and $\Gamma_{F_{4}}=\Gamma_{3}$. A regular octagon inscribed in the same circumference $\Gamma_{3}$ is instead represented in red

bers) such that

$G_{n}:=G_{n-2}+G_{n-1}$ for all $n \geq 2$

is said a generalized Fibonacci sequence, and the couple $\left(G_{0}, G_{1}\right)$ is called the seed of the sequence. For example, the generalized Fibonacci sequence with seed $(2,1)$ is called Lucas sequence and denoted by $\left\{L_{n}\right\}_{n \in \mathbb{N}_{0}}$. Hence, we have

$$
\begin{aligned}
& L_{0}=2, \quad L_{1}=1, \quad L_{2}=3, \quad L_{4}=4, \\
& L_{5}=7, \quad L_{6}=11, \quad L_{7}=18, \quad \text { etc. }
\end{aligned}
$$

Now, we begin to talk about words, i.e., finite or infinite sequences of symbols from a finite alphabet $A$, starting with some definitions that we need for our purposes.

For any real numbers $\beta, \rho$ with $\beta>0$, consider the line

$L: \quad y=\beta x+\rho$

in the plane $\mathbb{R}^{2}$, the grid $G:=\left\{(x, y) \in \mathbb{R}^{2}: x\right.$ or $y \in$ $\mathbb{Z}$, and $x \geq 0\}$ in the right half plane $H:=\left\{(x, y) \in \mathbb{R}^{2}\right.$ : $x \geq 0\}$, and the sequence of intersection points of $L \cap G$

$T_{0}=\left(x_{0}, y_{0}\right)=(0, \rho), \quad T_{1}=\left(x_{1}, y_{1}\right)$,

$T_{2}=\left(x_{2}, y_{2}\right), \quad T_{3}=\left(x_{3}, y_{3}\right), \quad$ etc.,

where $0=x_{0}<x_{1}<x_{2}<\ldots$ and $L \cap G=\left\{T_{n}: n \in \mathbb{N}_{0}\right\}$.
Definition 1 (i) From the sequence $\left\{T_{n}\right\}_{n \in \mathbb{N}_{0}}$, we define an infinite word $K_{\beta, \rho}$ by writing 0 for $T_{0}$ and continuing in succession for all $T_{n}, n \geq 1$, by writing

- 0 if $x_{n} \in \mathbb{Z}$ and $y_{n} \notin \mathbb{Z}$;

- 1 if $y_{n} \in \mathbb{Z}$ and $x_{n} \notin \mathbb{Z}$;

- 10 if $x_{n} \in \mathbb{Z}$ and $y_{n} \in \mathbb{Z}$ too.

$K_{\beta, \rho}$ is called the lower cutting sequence attached to the line $L$.

(ii) Similarly, we define an infinite word $K_{\beta, \rho}^{\prime}$ called the upper cutting sequence attached to the line $L$, by writing 1 if $y_{0} \in \mathbb{Z}$ or 0 if $y_{0} \notin \mathbb{Z}$, and continuing for all $T_{n}, n \geq 1$, by writing

- 0 if $x_{n} \in \mathbb{Z}$ and $y_{n} \notin \mathbb{Z}$;

- 1 if $y_{n} \in \mathbb{Z}$ and $x_{n} \notin \mathbb{Z}$;

- 01 if $x_{n}, y_{n} \in \mathbb{Z}$.

For the previous definitions, the reader can also see (Lothaire 2002, Remark 2.1.12). We now give a very easy example.

Example 3 Considering the line $y=2 x / 5$, we trivially find

$K_{2 / 5,0}=000100100010010001001 \ldots$

Hence, the lower cutting sequence relative to the line $y=$ $2 x / 5$ is an infinite periodic word with period 4 and periodic factor 0001001.

We are now ready to give the central definition of the section. Recalling the construction of the Carboncettus octagon $C_{n}$ in the previous section, let

$y=\beta_{n} x$

be the line through the origin and the point $B_{n}, n \geq 1$ (Fig. 3).

Definition 2 (The Carboncettus word $W_{n}$ ) For every $n \in \mathbb{N}$, we set

$W_{n}:=K_{\beta_{n}, 0}$

and we call $W_{n}$ the $n t h$ Carboncettus infinite word or, simply, the $n$th Carboncettus word.

Remark 2 Looking at Fig. 3, we can easily note that

$\left|O H_{n}\right|=\sqrt{\left|O B_{n}\right|^{2}-\left|H_{n} B_{n}\right|^{2}}=\sqrt{F_{n+2}^{2}-F_{n}^{2}} ;$

hence,

$\beta_{n}=\tan \left(\measuredangle H_{n} O B_{n}\right)=\frac{F_{n}}{\sqrt{F_{n+2}^{2}-F_{n}^{2}}}$

\footnotetext{
${ }^{4}$ If the reader want to read more on the notion of period for finite and infinite word, we refer him/her to Lothaire (2002).
} 
Table 1 Some metric data relative to the three Carboncettus octagons $C_{2}, C_{3}, C_{4}$, in the second, third and fourth column, respectively. Recall that the letters with the subscript $n$ are displayed in the general construction of $C_{n}$ shown in Fig. 3

\begin{tabular}{llll}
\hline & $n=2$ & $n=3$ & $n=4$ \\
\hline$\left|O K_{n}\right|$ & 1 & 2 & 3 \\
$\left|O A_{n}\right|$ & 3 & 5 & 8 \\
$\left|A_{n} B_{n}\right|$ & 2 & 4 & 6 \\
$\left|B_{n} D_{n}\right|$ & $4-\sqrt{2}$ & $\sqrt{42}-2 \sqrt{2}$ & $\sqrt{110}-3 \sqrt{2}$ \\
$\left|O H_{n}\right|$ & $2 \sqrt{2}$ & $\sqrt{21}$ & $\sqrt{55}$ \\
$\left|O N_{n}\right|$ & $2+\sqrt{2} / 2$ & $\sqrt{2}+\sqrt{42} / 2$ & $(3 \sqrt{2}+\sqrt{110}) / 2$ \\
$\measuredangle A_{n} O B_{n}$ & $\approx 38.942^{\circ}$ & $\approx 47.156^{\circ}$ & $\approx 44.049^{\circ}$ \\
$\measuredangle B_{n} O D_{n}$ & $\approx 51.058^{\circ}$ & $\approx 42.844^{\circ}$ & $\approx 45.951^{\circ}$ \\
$\measuredangle B_{n} A_{n} O$ & $\approx 70.529^{\circ}$ & $\approx 66.421^{\circ}$ & $\approx 67.976^{\circ}$ \\
$\operatorname{Perim}\left(C_{n}\right)$ & $24-4 \sqrt{2}$ & $16+4 \sqrt{42}-8 \sqrt{2}$ & $24+4 \sqrt{110}-12 \sqrt{2}$ \\
$\operatorname{Area}\left(C_{n}\right)$ & $14+8 \sqrt{2}$ & $34+8 \sqrt{21}$ & $92+12 \sqrt{55}$ \\
\hline
\end{tabular}

for all $n \in \mathbb{N}$.

Example 4 (The first Carboncettus word $W_{1}$ ) From Definition 2, the first Carboncettus word is the lower cutting sequence relative to the line obtained as extension of the segment $O B_{1}$ in Fig. 2. Using (8) or recalling from Example 1 that $\measuredangle H_{1} O B_{1}=\pi / 6$, we have

$\beta_{1}=\frac{1}{\sqrt{3}} \approx 0.577350269$

Then, we can easily compute the first digits of $W_{1}$ as follows:

$$
\begin{aligned}
W_{1}= & K_{1 / \sqrt{3}, 0} \\
= & 001001001010010010010100100100 \\
& 101001001010010010010100100100 \\
& 101001001001010010010100100100 \\
& 101001001001010010010010100100 \\
& 101001001001010010010010100 \ldots
\end{aligned}
$$

Example 5 (The Carboncettus words $W_{2}, W_{3}$ and $W_{4}$ )

(i) Using (8), we obtain

$$
\beta_{2}=\frac{F_{2}}{\sqrt{F_{4}^{2}-F_{2}^{2}}}=\frac{\sqrt{2}}{4} \approx 0.353553390 ;
$$

hence, by some simple computations, we find the first digits of the Carboncettus word $W_{2}$ as follows:

$$
\begin{aligned}
W_{2}= & K_{\sqrt{2} / 4,0} \\
= & 000100010001000100010010001000 \\
& 100010001000100100010001000100 \\
& 010001001000100010001000100010 \\
& 010001000100010001000100100010 \\
& 001000100010010001000100010 \ldots
\end{aligned}
$$

(ii) Using (8) as before, we find

$$
\beta_{3}=\frac{F_{3}}{\sqrt{F_{5}^{2}-F_{3}^{2}}}=\frac{2 \sqrt{21}}{21} \approx 0.436435780
$$

and, by simple computations, we get the first digits of the Carboncettus word $W_{3}$ as follows:

$$
\begin{aligned}
W_{3}= & K_{2 \sqrt{21} / 21,0} \\
= & 000100100100010010010001001001 \\
& 001000100100100010010010010001 \\
& 001001000100100100100010010010 \\
& 001001001000100100100100010010 \\
& 010001001001001000100100100 \ldots
\end{aligned}
$$

(iii) Using (8) as in (i) and (ii), we get

$$
\beta_{4}=\frac{F_{4}}{\sqrt{F_{6}^{2}-F_{4}^{2}}}=\frac{3 \sqrt{55}}{55} \approx 0.404519917
$$

and then the following first 147 digits of the Carboncettus word $W_{4}$

$$
\begin{aligned}
W_{4}= & K_{3 \sqrt{55} / 55,0} \\
= & 000100100010010001001000100100 \\
& 010010001001000100100010010001 \\
& 001001000100100010010001001000 \\
& 100100010010001001000100100010 \\
& 010010001001000100100010010 \ldots
\end{aligned}
$$

The definition of mechanical word or mechanical sequence is similar to Definition 1. As usual, we let $\lfloor x\rfloor$ and $\lceil x\rceil$ denote, respectively, the floor and the ceiling of a real number $x$. 
Definition 3 For any $\alpha, \rho \in \mathbb{R}$ with $0 \leq \alpha \leq 1$, we define two infinite words

$s_{\alpha, \rho}: \mathbb{N}_{0} \longrightarrow\{0,1\}, \quad s_{\alpha, \rho}^{\prime}: \mathbb{N}_{0} \longrightarrow\{0,1\}$

by setting

$s_{\alpha, \rho}(n):=\lfloor\alpha(n+1)+\rho\rfloor-\lfloor\alpha n+\rho\rfloor$

and

$s_{\alpha, \rho}^{\prime}(n):=\lceil\alpha(n+1)+\rho\rceil-\lceil\alpha n+\rho\rceil$

for all $n \in \mathbb{N}_{0}$. The word $s_{\alpha, \rho}\left[s_{\alpha, \rho}^{\prime}\right.$, respectively $]$ is called the lower [upper, resp.] mechanical word with slope $\alpha$ and intercept $\rho$. Moreover, a mechanical word $s_{\alpha, \rho}$ or $s_{\alpha, \rho}^{\prime}$ is said rational if $\alpha \in \mathbb{Q}$ and irrational if $\alpha \notin \mathbb{Q}$.

There are several equivalent definitions of Sturmian word related to different properties, for which we refer to (Lothaire 2002, Section 2). The simplest way to define it is the following.

Definition 4 A Sturmian word is an infinite word $w$ over an alphabet $A$ which contains exactly $n+1$ factors of length $n$ for all $n \in \mathbb{N}_{0}$.

For instance, considering factors of length 1, the definition above implies that every Sturmian word must necessarily be over two letters of $A$, i.e., a binary word. The following theorem establishes which Carboncettus words are Sturmian.

Theorem 1 All Carboncettus words $W_{n}$ are Sturmian words except when $n=5$; in this case, we have an infinite purely periodic word with period 17 and periodic factor 00010010001001001.

Proof rom Theorem 2.1.13 of Lothaire (2002), a lower mechanical word $s_{\alpha, \rho}$ is a Sturmian word if and only if it is irrational or, equivalently, if and only if the slope $\alpha$ is irrational. The Carboncettus word $W_{n}$ is equal to $K_{\beta_{n}, 0}$ by definition, and it is well known and easy to prove that a lower cutting sequence $K_{\beta, \rho}$ is equal to the lower mechanical word obtained by "dividing the couple $(\beta, \rho)$ by $\beta+1$," that is, in symbols

$K_{\beta, \rho}=s_{\beta /(\beta+1), \rho /(\beta+1)}$

(see, for instance, Lothaire 2002, Section 2.1.2). Since $\beta /(\beta+1)=1-1 /(\beta+1)$, then $\beta /(\beta+1)$ is irrational if and only if $\beta$ itself is irrational. In conclusion, we have

$W_{n}=s_{\beta_{n} /\left(\beta_{n}+1\right), 0}$ is Sturmian $\Leftrightarrow \beta_{n} \notin \mathbb{Q}$.
Now, from Remark 2 we have

$$
\begin{aligned}
\beta_{n} & =\frac{F_{n}}{\sqrt{F_{n+2}^{2}-F_{n}^{2}}} \\
& =\frac{F_{n}}{\sqrt{\left(F_{n+2}-F_{n}\right)\left(F_{n+2}+F_{n}\right)}} \\
& =\frac{F_{n}}{\sqrt{F_{n+1}\left(F_{n+2}+F_{n}\right)}}
\end{aligned}
$$

for all $n \in \mathbb{N}$. By an induction argument on $n$, it is immediate to prove that

$F_{n+m}=F_{n} \cdot F_{m-1}+F_{n+1} \cdot F_{m}$

for all $n \geq 0, m \geq 1$ : For this purpose, consider $m \geq 1$ fixed and assume that (15) is true for $n=k$ and $n=k+1$. Adding side to side, (15) is hence true for $n=k+2$, and since it trivially holds for $n=0$ and $n=1,(15)$ is proved in general.

Using (15) with $m=n+2$, we then get from (14)

$\beta_{n}=\frac{F_{n}}{\sqrt{F_{n} F_{n+1}+F_{n+1} F_{n+2}}}=\frac{F_{n}}{\sqrt{F_{2 n+2}}}$

for all $n \geq 1 ;^{5}$ hence, $\beta_{n}$ is rational if and only if $F_{2 n+2}$ is a perfect square. But Cohn and Wyler proved independently in 1964 that a Fibonacci number $F_{m}, m \in \mathbb{N}_{0}$, is a square if and only if $m=0,1,2,12$ (see Cohn 1964 and Wyler and Rollett (1964)); hence,

$F_{2 n+2}, n \geq 1$, is a square $\Leftrightarrow n=5$.

Therefore, (13), (16) and (17) together prove the first part of the thesis.

If $n=5$, we get from (8)

$\beta_{5}=\frac{F_{5}}{\sqrt{F_{7}^{2}-F_{5}^{2}}}=\frac{5}{12}$,

and considering the line $y=5 x / 12$, by some trivial computations, we conclude that $W_{5}=K_{5 / 12,0}$ is a purely periodic word with period $5+12=17$ and periodic factor 00010010001001001 , i.e.,

$$
\begin{aligned}
& W_{5}=0001001000100100100010010001001001 \\
& 00010010001001001 \ldots
\end{aligned}
$$

\footnotetext{
$\overline{5 \text { To obtain (16) }}$, the more expert reader could quickly observe that
} $F_{n+1}\left(F_{n+2}+F_{n}\right)=F_{n+1} L_{n+1}=F_{2(n+1)}$. 
In the next example, we compute the first digits of three more words after the special case $W_{5}$. We will also need these explicit determinations in Example 8 of the next section.

Example 6 (The words $W_{6}, W_{7}$ and $W_{8}$ )

(i) Using (8), we obtain

$$
\beta_{6}=\frac{F_{6}}{\sqrt{F_{8}^{2}-F_{6}^{2}}}=\frac{8 \sqrt{377}}{377} \approx 0.412020962 ;
$$

hence, as in Example 5, we find the first 147 digits of the Carboncettus word $W_{6}$ as follows:

$$
\begin{aligned}
W_{6}= & K_{8 \sqrt{377} / 377,0} \\
= & 000100100010010001001001000100 \\
& 100010010001001001000100100010 \\
& 010001001001000100100010010001 \\
& 001001000100100010010001001001 \\
& 000100100010010001001001000 \ldots
\end{aligned}
$$

(ii) For $W_{7}$, we find

$$
\beta_{7}=\frac{F_{7}}{\sqrt{F_{9}^{2}-F_{7}^{2}}}=\frac{13 \sqrt{987}}{987} \approx 0.413794559
$$

and then we get the following first 147 digits

$$
\begin{aligned}
W_{7}= & K_{13 \sqrt{987} / 987,0} \\
= & 000100100010010001001001000100 \\
& 100010010010001001000100100010 \\
& 010010001001000100100100010010 \\
& 001001000100100100010010001001 \\
& 001000100100010010001001001 \ldots
\end{aligned}
$$

(iii) For $W_{8}$, we find

$$
\beta_{8}=\frac{F_{8}}{\sqrt{F_{10}^{2}-F_{8}^{2}}}=\frac{21 \sqrt{646}}{1292} \approx 0.413116974
$$

and, consequently,

$$
\begin{aligned}
W_{8}= & K_{21 \sqrt{646} / 1292,0} \\
= & 000100100010010001001001000100 \\
& 100010010001001001000100100010 \\
& 010010001001000100100010010010 \\
& 001001000100100010010010001001 \\
& 000100100100010010001001000100 \\
& 100100010010001001000100100 \ldots
\end{aligned}
$$

Note that for $W_{8}$ we have written 30 more digits (177) than for the previous words: The reason for this will be clear later.

\section{The Carboncettus limit word}

If $A$ is a finite alphabet, the set of right infinite words over $A$ is usually denoted by $A^{\mathbb{N}_{0}}$ or $A^{\omega}$. It is equipped with a distance $d$ defined as follows: For any

$x=x_{0} x_{1} \ldots x_{k} \ldots$ and $y=y_{0} y_{1} \ldots y_{k} \ldots$

belonging to $A^{\omega}$, we set

$d(x, y):= \begin{cases}2^{-\min \left\{k \in \mathbb{N}_{0}: x_{k} \neq y_{k}\right\}} & \text { if } x \neq y ; \\ 0 & \text { if } x=y .\end{cases}$

In this way, $\left(A^{\omega}, d\right)$ is a compact metric space (hence complete and totally bounded by a fundamental result in general topology) called the Cantor space (see Lothaire 2002, Chap. 1). From the distance defined in (23), it follows that a sequence of words $\left\{X_{n}\right\}_{n} \subset A^{\omega}$ converges to $Y \in A^{\omega}$, and we write

$\lim _{n \rightarrow \infty} X_{n}=Y$

in this case, if, for every $i \in \mathbb{N}_{0}$, we have $\left(X_{n}\right)_{i}=Y_{i}$ for all sufficiently large $n$, i.e., greater than some $v(i)$. In agreement with (22), the previous notations

$\left(X_{n}\right)_{i}$ and $Y_{i}$

obviously indicate the letter of the words $X_{n}$ and $Y$, respectively, associated with $i$.

Now that we have the notations and definitions necessary for this section, let us reconsider Fibonacci numbers. Recalling that the sequence of ratios of two consecutive Fibonacci numbers $\left\{F_{n+1} / F_{n}\right\}_{n \in \mathbb{N}_{0}}$ converges to the golden section $\phi=(1+\sqrt{5}) / 2,{ }^{6}$ then

\footnotetext{
${ }^{6}$ Since in the Introduction we are committed to making the paper selfcontained (with the exception of Theorem 1), we sketch here what we think is the most elementary proof of $\lim _{n \rightarrow \infty} F_{n+1} / F_{n}=\phi$. Using only definition (1) and identity (26), it is in fact immediate to prove, through a trivial induction argument, that
}

$$
\left|F_{n+1} / F_{n}-\phi\right|<1 / \phi^{n-1}
$$

for all $n \geq 1$. For the inductive step note that, if $n-1 \geq 1$, then

$$
\begin{aligned}
\left|\frac{F_{n+1}}{F_{n}}-\phi\right| & =\left|\frac{F_{n-1}+F_{n}}{F_{n}}-\frac{\phi^{2}}{\phi}\right|=\left|\frac{F_{n-1}}{F_{n}}-\frac{1}{\phi}\right| \\
& =\frac{1}{F_{n} \phi / F_{n-1}} \cdot\left|\frac{F_{n}}{F_{n-1}}-\phi\right|<\frac{1}{\phi} \cdot \frac{1}{\phi^{n-2}}=\frac{1}{\phi^{n-1}},
\end{aligned}
$$




$$
\begin{aligned}
\lim _{n \rightarrow \infty} \frac{F_{n+2}^{2}-F_{n}^{2}}{F_{n}^{2}} & =\lim _{n \rightarrow \infty}\left(\frac{F_{n+2}}{F_{n}}\right)^{2}-1 \\
& =\lim _{n \rightarrow \infty}\left(\frac{F_{n+2}}{F_{n+1}} \cdot \frac{F_{n+1}}{F_{n}}\right)^{2}-1 \\
& =\left(\phi^{2}\right)^{2}-1 \\
& =3 \phi+1,
\end{aligned}
$$

where in the last step we used the equality

$\phi^{2}=\phi+1$.

Recalling (8), we then define

$$
\begin{aligned}
\beta_{\infty} & :=\lim _{n \rightarrow \infty} \beta_{n}=\lim _{n \rightarrow \infty} \frac{F_{n}}{\sqrt{F_{n+2}^{2}-F_{n}^{2}}} \\
& =\lim _{n \rightarrow \infty}\left(\sqrt{\frac{F_{n+2}^{2}-F_{n}^{2}}{F_{n}^{2}}}\right)^{-1}
\end{aligned}
$$

and, using (25) and (26), we obtain

$$
\begin{aligned}
\beta_{\infty} & =(\sqrt{3 \phi+1})^{-1}=\sqrt{\frac{1}{3 \phi+1} \cdot \frac{3 \phi-4}{3 \phi-4}} \\
& =\sqrt{\frac{3 \phi-4}{5}}=\sqrt{\frac{3 \sqrt{5}-5}{10}} \\
& \approx 0.4133042381 .
\end{aligned}
$$

This leads to consider the line

$y=\beta_{\infty} x$

and to state the following:

Definition 5 Similar to Definition 2, we set

$W_{\infty}:=K_{\beta_{\infty}, 0}=K \sqrt{(3 \sqrt{5}-5) / 10}, 0$

and we call $W_{\infty}$ the Carboncettus limit word.

Footnote 6 continued

where to obtain the previous inequality we used $F_{n} \phi / F_{n-1} \geq \phi$ and the inductive hypothesis.
The first digits of $W_{\infty}$ can be easily computed as in Examples 4,5 and 6 , getting

$$
\begin{aligned}
W_{\infty}= & K \sqrt{(3 \sqrt{5}-5) / 10}, 0 \\
= & 000100100010010001001001000100 \\
& 100010010001001001000100100010 \\
& 010010001001000100100010010010 \\
& 001001000100100010010010001001 \\
& 000100100100010010001001000100 \\
& 100100010010001001001000100 \ldots
\end{aligned}
$$

Consider now a family, or better, a sequence of lines $\left\{L_{n}\right.$ : $n \in \mathbb{N}\}$ with positive slopes in the plane $\mathbb{R}^{2}$, and assume that it "converges" to some line $L$ of the form (5). Then, it is not true in general that the cutting sequence (lower or upper) attached to $L_{n}$ approaches the cutting sequence attached to $L$ in the sense of the definition given in (24). And the same can be said for the slopes of a sequence of mechanical or balanced words. The following is a very simple example of what can happen.

Example 7 For any $n \in \mathbb{N}$, consider the line

$$
L_{n}: \quad y=\left(\frac{2}{5}-\frac{1}{n}\right) x
$$

through the origin of the plane. It is clear that the sequence $\left\{L_{n}\right\}_{n \in \mathbb{N}}$ converges (punctually) to the line $L: y=$ $2 x / 5$ considered in Example 3, but the sequence of words $\left\{K_{2 / 5-1 / n, 0}\right\}_{n \in \mathbb{N}}$ does not converge to $K_{2 / 5,0}$ shown in (6). In fact, it is simple to check that, for every $n \geq 15$, the first nine digits of $K_{2 / 5-1 / n, 0}$ are 000100010 , hence different from the ones of $K_{2 / 5,0}$.

We are now ready to prove the main result of the section.

Theorem 2 The limit of the sequence of Carboncettus words is the Carboncettus limit word, in symbols

$\lim _{n \rightarrow \infty} W_{n}=W_{\infty}$

Proof We want to show that for each fixed $i \in \mathbb{N}_{0}$ it holds

$\left(W_{n}\right)_{i}=\left(W_{\infty}\right)_{i}$

for all $n \in \mathbb{N}$ large enough. Therefore, assume by reductio ad absurdum that this is not true for all $i$, and let $j$ be the smallest $i$ such that (32) is not satisfied for infinitely many $n \in \mathbb{N}$. Then, for each positive integer $m \leq j$ consider the following unitary open interval

]$\left\lfloor\beta_{\infty} \cdot m\right\rfloor,\left\lceil\beta_{\infty} \cdot m\right\rceil[$ 
and since $\beta_{n} \rightarrow \beta_{\infty}$ by definition and $\beta_{\infty} \notin \mathbb{Q}$ (recall (27) and (28), resp.), then there exists $v \in \mathbb{N}$ such that

$\left.\beta_{n} \cdot m \in\right]\left\lfloor\beta_{\infty} \cdot m\right\rfloor,\left\lceil\beta_{\infty} \cdot m\right\rceil[$

for all $n \geq v$ and $m \leq j$. Now, recalling the definition of lower cutting sequence (see Definition 1(i)), this means that for every integer $i$ from 0 to at least $j$ we have $\left(W_{n}\right)_{i}=$ $\left(W_{\infty}\right)_{i}$ for all $n \geq v$, and this contradicts our assumption.

The previous theorem claims that the sequence of words whose first elements are (9)-(12), (18) and (19)-(21) converges to (31). Using the definition of distance given in (23), it is possible to define a further sequence

$\left\{\delta_{n}\right\}_{n \in \mathbb{N}} \quad$ where $\quad \delta_{n}:=d\left(W_{n}, W_{\infty}\right)$.

Example 8 Numerically, the first elements of $\left\{\delta_{n}\right\}_{n}$ are the following:

$\delta_{1}=2^{-2}, \quad \delta_{2}=2^{-6}$,

$\delta_{3}=2^{-9}, \quad \delta_{4}=2^{-23}$,

$\delta_{5}=2^{-16}, \quad \delta_{6}=2^{-64}$,

$\delta_{7}=2^{-40}, \quad \delta_{8}=2^{-170}$,

as the reader can immediately verify comparing (9)-(12) and (18)-(21) with (31).

\section{Conclusion and future work}

After the introductive section, to move faster to present Carboncettus words, we have reduced to a minimum an important geometric section like the second one. In a later work (on "the Prato octagon and regularity measures for polygons," see Caldarola 2020), we will devote more space to the sequence $\left\{C_{n}\right\}_{n}$, studying in more depth the characteristics of its elements. For example, already in Caldarola et al. (2020c) there was mention of the existence of the "limit normalized octagon" $C_{\infty}^{N}$, in analogy with $W_{\infty}$. Since the circumferences $\Gamma_{F_{n}}$ and $\Gamma_{F_{n+2}}$ become larger and larger, $C_{\infty}^{N}$ is obtained by normalizing the radius of the internal one. Another approach to study the limit of $\left\{C_{n}\right\}_{n}$ without carrying out normalizations, say $C_{\infty}$, is to use non-standard mathematics or a numerical system that allows calculations with infinite numbers. ${ }^{7}$

\footnotetext{
${ }^{7}$ Among many non-standard settings useful for this purpose, the simplest is probably the computational one described in (Sergeyev 2003, 2007, 2017) and also used in (Antoniotti et al. 2020a,b; Caldarola 2018b, a; Caldarola et al. 2020a, e) with limit curves and polytopes, fractals, or with speculative-didactic intentions. Such computational system is even applied to Fibonacci words in Margenstern (2015).
}

We find it fascinating to be able to construct regular or almost regular geometric figures, by means of simple or approximate methods, such as the one shown here which uses Fibonacci numbers to obtain a family of octagons that are indistinguishable from a regular one. And this must be understood not only limited to the figures of the Euclidean plane. The interest, moreover, is undoubtedly greater if it is possible to trace ancient uses or historical foundations of approximate methods, very rare until modern times, as far as we know today.

Much work remains to be done also on the purely combinatorial-algebraic aspects highlighted here, such as the sequence of Carboncettus words and its properties, similar or derived sequences from $\left\{C_{n}\right\}_{n}$, the characteristics of the elements in $\left\{C_{n}\right\}_{n}$, etc. For example, the sequence $\left\{\delta_{n}\right\}_{n}$ defined in (33) does not decrease monotonously as it is immediately evident from (34), but it is easy to prove that the two subsequences of odd- and even-indexed terms, i.e., $\left\{\delta_{2 n+1}\right\}_{n}$ and $\left\{\delta_{2 n}\right\}_{n}$, respectively, are monotone (this is because they depend on the approach of the line (7) to (29) from the above and below, resp.). Thus, it seems interesting to study and compare their speeds of convergence to zero and other aspects also in perspective of the so-called unimaginable numbers.

The unimaginable numbers are numbers so large that they cannot be written through the ordinary scientific or exponential notation, but they need notational systems specifically designed for the purpose, such as Knuth up-arrow notation, Conway chained arrow notation, Steinhaus-Moser notation, Bowers's operators and others (see Caldarola et al. 2020b, d and the references therein for more details). The usual convention is that an unimaginable number is a number greater than 1 googol $=10^{100} .8$

In a sequence of converging words, it often happens that the distances decrease very quickly and become very soon "unimaginably small." For instance, for the fourth element of the sequence $\left\{\delta_{2 n}\right\}_{n}$, corresponding to the fourth line of the form (7) from the above, we already have

$\delta_{8}=d\left(W_{8}, W_{\infty}\right)=2^{-170} \approx \frac{6.682}{10^{52}}<\frac{1}{\sqrt{1 \text { googol }}}$,

and, more importantly, note the rapid growth of the exponents in the right column in (34). Unfortunately, however, despite various evidences, many links between combinatorics of words and unimaginable numbers remain almost unexplored nowadays, and we hope that many researchers,

\footnotetext{
$\overline{8}$ The number 1 googol is in fact a rather significative threshold for several reasons, among them because it is of the order of magnitude of the greatest numbers in the physical realm. For example, the Eddington number $N_{\text {Edd }}$, which represents the number of protons (or electrons) in the observable universe, should be about $10^{80}$, and the total volume of the observable universe itself should be about $10^{128}$ times that of a neutron.
} 
from different parts of the world, will soon be interested in systematically studying these fascinating topics.

Acknowledgements This work was partially supported by the research projects “IoT\& B, Internet of Things and Block-chain", CUP J48C 17000 230006, POR Calabria FESR-FSE 2014-2020. The authors are indebted to the anonymous reviewers who, with their meticulous observations, have allowed them to significantly improve the readability of this paper.

Funding Open access funding provided by Università della Calabria within the CRUI-CARE Agreement.

\section{Compliance with ethical standards}

Conflict of interest The authors declare that they have no conflict of interest.

Open Access This article is licensed under a Creative Commons Attribution 4.0 International License, which permits use, sharing, adaptation, distribution and reproduction in any medium or format, as long as you give appropriate credit to the original author(s) and the source, provide a link to the Creative Commons licence, and indicate if changes were made. The images or other third party material in this article are included in the article's Creative Commons licence, unless indicated otherwise in a credit line to the material. If material is not included in the article's Creative Commons licence and your intended use is not permitted by statutory regulation or exceeds the permitted use, you will need to obtain permission directly from the copyright holder. To view a copy of this licence, visit http://creativecomm ons.org/licenses/by/4.0/.

\section{References}

Albano A (2015) The Fibonacci sequence and the golden section in a lunette decoration of the medieval church of San Nicola in Pisa. Territori della Cultura 21:48-59

Antoniotti A, Caldarola F, d'Atri G, Pellegrini M (2020a) New approaches to basic calculus: An experimentation via numerical computation. In: Sergeyev YD, Kvasov DE (eds) Proceedings of the 3rd International Conference "Numerical Computations: Theory and Algorithms". LNCS, vol 11973, pp 329-342. Springer, Cham (2020). https://doi.org/10.1007/978-3-030-39081-5_29

Antoniotti L, Caldarola F, Maiolo M (2020b) Infinite numerical computing applied to Peano's, Hilbert's, and Moore's curves. Mediterranean J Math 17:99. https://doi.org/10.1007/s00009-020-015315

Armienti P (2016) The medieval roots of the modern scientific thought. A Fibonacci abacus on the facade of the church of San Nicola in Pisa. J Cultural Herit 17:1-6

Berstel J, Perrin D (2007) The origins of combinatorics on words. Eur J Comb 28:996-1022. https://doi.org/10.1016/j.ejc.2005.07.019

Caldarola F (2018) The exact measures of the Sierpiński $d$-dimensional tetrahedron in connection with a Diophantine nonlinear system. Commun Nonlinear Sci Numer Simul 63:228-238. https://doi.org/ 10.1016/j.cnsns.2018.02.026

Caldarola F (2018b) The Sierpiński curve viewed by numerical computations with infinities and infinitesimals. Appl Math Comput 318:321-328. https://doi.org/10.1016/j.amc.2017.06.024
Caldarola, F (2020) The Prato octagon: from Fibonacci numbers to regularity measures for polygons (2020). Preprint/work in progress

Caldarola F, Cortese D, d'Atri G, Maiolo M (2020a) Paradoxes of the infinite and ontological dilemmas between ancient philosophy and modern mathematical solutions. In: Sergeyev YD, Kvasov D (eds) Proceedings of the 3rd International Conference "Numerical Computations: Theory and Algorithms". LNCS, vol 11973, pp 358-372. Springer, Cham (2020). https://doi.org/10.1007/978-3030-39081-5_31

Caldarola F, d'Atri G, Maiolo M (2020b) What are the "unimaginable numbers"? In: Caldarola F, d'Atri G, Maiolo M, Pirillo G (eds.) Proceedings of the International Conference "From Pythagoras to Schützenberger". Pellegrini Editore, Cosenza (IT) (2020 (in print))

Caldarola F, d'Atri G, Maiolo M, Pirillo G (2020c) The sequence of Carboncettus octagons. In: Sergeyev YD, Kvasov D (eds) Proceedings of the 3rd International Conference "Numerical Computations: Theory and Algorithms", LNCS, vol 11973, pp 373-380. Springer, Cham (2020). https://doi.org/10.1007/978-3-030-39081-5_32

Caldarola F, d'Atri G, Mercuri P, Talamanca V (2020d) On the arithmetic of Knuth's powers and some computational results about their density. In: Sergeyev YD, Kvasov D (eds) Proceedings of the 3rd International Conference "Numerical Computations: Theory and Algorithms", LNCS, vol 11973, pp 381-388. Springer, Cham (2020). https://doi.org/10.1007/978-3-030-39081-5_33

Caldarola F, Maiolo M, Solferino V (2020e) A new approach to the Ztransform through infinite computation. Commun Nonlinear Sci Numer Simul. 82:105019. https://doi.org/10.1016/j.cnsns.2019. 105019

Cohn JHE (1964) On square Fibonacci numbers. J London Math Soc 39:537-540

Lothaire M (1983) Combinatorics on words, Encyclopedia of mathematics and its applications, vol 17. Addison-Wesley, Reading

Lothaire M (2002) Algebraic combinatorics on words, Encyclopedia of mathematics and its applications, vol 90. Cambridge University Press, Cambridge

Margenstern M (2015) Fibonacci words, hyperbolic tilings and grossone. Commun Nonlinear Sci Numer Simul 21:3-11

Pirillo G (2017) Figure geometriche su un portale del Duomo di Prato. Prato Storia e Arte 121:7-16

Pirillo G (2017) La scuola pitagorica ed i numeri di Fibonacci. Archimede 2:66-71

Pirillo G (2017) L'origine pitagorica dei numeri di Fibonacci. Periodico di Matematiche 9:99-103

Pirillo G (2018) A characterization of Fibonacci numbers. Chebyshevskii Sbornik 19:259-271

Sergeyev YD (2003) Arithmetic of infinity. Edizioni Orizzonti Meridionali, Cosenza

Sergeyev YD (2007) Blinking fractals and their quantitative analysis using infinite and infinitesimal numbers. Chaos Solitons Fractals 33(1):50-75

Sergeyev YD (2017) Numerical infinities and infinitesimals: methodology, applications, and repercussions on two Hilbert problems. EMS Surv Math Sci 4:219-320

Wyler O, Rollett AP (1964) Squares in the Fibonacci series. Advanced problems and solutions: solution: 5080. Am Math Month 71:220 222

Publisher's Note Springer Nature remains neutral with regard to jurisdictional claims in published maps and institutional affiliations. 